\title{
Astrophysical constraints on a possible neutrino ball at the Galactic Center
}

\author{
F. De Paolis ${ }^{1}$, G. Ingrosso ${ }^{1}$, A. A. Nucita ${ }^{1}$, D. Orlando ${ }^{1}$, S. Capozziello ${ }^{2}$, and G. Iovane ${ }^{2}$ \\ 1 Dipartimento di Fisica, Università di Lecce, and INFN, Sezione di Lecce, Via Arnesano, CP 193, \\ 73100 Lecce, Italy \\ 2 Dipartimento di Fisica "E. R. Caianiello", Università di Salerno, and INFN, Sezione di Salerno, Via S. Allende, \\ 84081 Baronissi (Sa), Italy
}

Received 7 February 2001 / Accepted 27 June 2001

\begin{abstract}
The nature of the massive object at the Galactic Center (Sgr A*) is still unclear even if various observational campaigns led many authors to believe that our Galaxy hosts a super-massive black hole with mass $M \simeq 2.6 \times 10^{6} M_{\odot}$. However, the black hole hypothesis, which theoretically implies a luminosity $\simeq 10^{41} \mathrm{erg} \mathrm{s}^{-1}$, runs into problems if one takes into account that the observed luminosity, from radio to $\gamma$-ray wavelengths, is below $10^{37} \mathrm{erg} \mathrm{s}^{-1}$. In order to solve this blackness problem, alternative models have recently been proposed. In particular, it has been suggested that the Galactic Center hosts a ball made up of non-baryonic matter (e.g. massive neutrinos and anti-neutrinos) in which the degeneracy pressure of fermions balances their self-gravity. Requiring it to be consistent with all the available observations towards the Galactic Center allows us to put severe astrophysical constraints on the neutrino ball parameters. The presence of such an object in the Galactic Center may be excluded if the constituent neutrino mass $m_{\nu}$ is $\gtrsim 24 \mathrm{keV}$, while if $m_{\nu} \lesssim 24 \mathrm{keV}$ observations cannot give a definite answer.
\end{abstract}

Key words. elementary particles - gravitation - Galaxy Center

\section{Introduction}

There is much evidence for the presence of super-massive black holes (SBHs) with masses in the range $10^{6}-10^{9} M_{\odot}$ in QSOs, AGNs and centers of galaxies. In our Galaxy, the discovery of the unusual radio source $\mathrm{SgrA}^{*}$ and the detailed information coming from star dynamics as led many authors to believe that our Galaxy also hosts a SBH with mass $(2.6 \pm 0.4) \times 10^{6} M_{\odot}$ (Genzel et al. 1996). Since this SBH should accrete the surrounding gas at a rate of $\dot{M} \simeq 10^{-6} M_{\odot} \mathrm{yr}^{-1}$ (Ghez et al. 1998), the usual radiative efficiency $\epsilon \simeq 0.1$ would imply a luminosity $L \geq 10^{41} \mathrm{erg} \mathrm{s}^{-1}$. However, the observed luminosity from radio to $\gamma$-ray wavelengths is below $10^{37} \mathrm{erg} \mathrm{s}^{-1}$, thereby implying that the SBH hypothesis runs into problems (Goldwurm et al. 1994). This is the so called "blackness problem" or the "black hole on starvation". Several alternative models have been proposed to solve the issue. For example, Narayan et al. (1998) proposed the advection dominated accretion flow model (ADAF), according to which most of the dissipated energy is channeled into protons that cannot radiate efficiently. However, as has

Send offprint requests to: F. De Paolis, e-mail: Francesco.DePaolis@le.infn.it been recently noted, the polarized radiation from SgrA* requires a nonthermal electron distribution for the emitting plasma and this seems to imply that the ADAF model is ruled out (Agol 2000). In principle, the direct observation of a mass density profile $\rho(r) \propto r^{-7 / 4}$ (Binney \& Tremaine 1987) very close to SgrA* should allow us to confirm the presence of a compact object like a SBH at the Galactic Center. However, the present observational techniques do not permit one to distinguish stars at distances $r \lesssim 10^{-2}$ pc from the Galactic Center, so that the SBH hypothesis at the Galaxy Center is far from being conclusive $^{1}$.

Recently Torres et al. (2000) have investigated the hypothesis that the Galactic Center could consist of a supermassive boson star. They analyzed stability configurations and dynamics, giving the prospects for the observational detection of such an object using the new generation of $\mathrm{X}$-ray, radio interferometry satellites and, in general, tools capable of detecting strong gravitational lensing effects. The conclusions were that the SBH hypothesis is, again, far from being definitive while the "signature" of boson

\footnotetext{
${ }^{1}$ For different methods to investigate the nature of the Galactic Center see e.g. De Paolis et al. (1996), De Paolis et al. (2001), and references therein.
} 
stars could be (or not) soon available in the case of very massive bosons (see e.g. Capozziello et al. 2000).

An alternative model to the SBH scenario at the Galactic Center has been proposed by some authors (Tsiklauri \& Viollier 1998a; Tsiklauri \& Viollier 1998b; Capozziello \& Iovane 1999). According to this scenario, neutrinos and anti-neutrinos could gravitationally interact forming super-massive neutrino balls in which the degeneracy pressure of fermions balances the self-gravity of the system.

Choosing neutrinos in a particular mass range implies the formation of super-massive fully degenerate objects with mass $\simeq 10^{6} M_{\odot}$. In particular, Capozziello \& Iovane (1999) proposed to investigate the eventual neutrino ball at the Galactic Center by gravitational lensing, since the neutrino ball acts as a transparent medium.

On the other hand, the existence of a neutrino ball at the Galactic Center avoids invoking the presence of a $\mathrm{SBH}$ and, under certain circumstances, should be able to justify the low luminosity (from radio to $\gamma$-rays) observed towards SgrA*.

However, if a neutrino ball really exists at the center of the Galaxy, this possibility must be consistent both with the theoretical mass limit of a stable configuration of fermions and with the currently available observational data, i.e. $i)$ the star dynamics within about one pc from $\mathrm{SgrA}^{*}$, ii) the low source luminosity. In addition, the interaction among neutrinos and anti-neutrinos within the ball, or the decay of neutrinos into neutrinos of different flavors, may also produce characteristic signatures revealing the object at the Galactic Center.

The aim of the present paper is to derive astrophysical constraints on the parameters of the neutrino ball with particular attention to the neutrino mass $m_{\nu}$. To this purpose, we relax the assumption of fully degenerate neutrino configurations adopted until now in the literature. Accordingly, we adopt a formalism based on the distribution function in phase-space allowing us to obtain more general fermion configurations with a degeneracy degree depending on the radial coordinate within the ball. In this formalism, either classical configurations (in which particles obey the Maxwellian statistics) and fully degenerate systems are naturally included. In this way, considering in addition the astrophysical constraints $i$ ) and ii) in the paragraph above, the allowed neutrino mass range turns out to be $11 \mathrm{keV} \lesssim m_{\nu} \lesssim 24 \mathrm{keV}$.

We note that assuming neutrinos in this mass range, it is also possible to build up neutrino ball models with total mass up to $10^{9}-10^{10} M_{\odot}$ and radius $\simeq 10^{-3}-10^{-2} \mathrm{pc}$. These objects might influence the accretion process of super-massive black holes in the AGN cores or completely mimic the central black holes, acting as the engine of AGNs. This possibility has been explored in some details by Tsiklauri \& Viollier (1996), but considering only fully degenerate self-gravitating configurations.

A further problem to be addressed is the cosmological implications of the existence of such heavy neutrinos. This problem has been discussed by several authors (e.g.
Kolb \& Turner 1990; Viollier 1994; Lindebaum et al. 1999; Dolgov \& Hansen 2000) to whom we refer for further details. Here we mention that an active neutrino $\left(\nu_{\mathrm{e}}, \nu_{\mu}\right.$ or $\nu_{\tau}$ ) of mass of a few $\mathrm{keV}$ is the warm dark matter candidate preferred by many authors, on the basis of $N$-body simulations of large scale structure formation (e.g. Colin et al. 2000). Indeed, Big Bang nucleosynthesis can only exclude active neutrino masses bigger than about $300 \mathrm{keV}$ (Dolgov et al. 1998). However, in the framework of the standard cosmology, active neutrinos with mass in the range $11 \mathrm{keV} \lesssim m_{\nu} \lesssim 24 \mathrm{keV}$, overclose the universe by a factor of about 100 (Kolb \& Turner 1990). Consequently, if heavy neutrinos formed and were in equilibrium in the early universe, they have to rapidly decay in order to not overclose it. Therefore, standard cosmology strongly constrains the presence of heavy neutrinos nowadays. Many authors have discussed this issue in the framework of more exotic cosmological scenarios. Indeed, it has been shown that the cosmological bound on neutrino mass can be bypassed in at least three ways: by $i$ ) avoiding thermalization of massive neutrinos with a reheat temperature (Giudice et al. 2000), ii) decay of neutrinos, iii) annihilation of neutrinos and anti-neutrinos (Bilić et al. 1998 and references therein). Another possibility has been explored by Dolgov \& Hansen (2000) who have shown that in the framework of a slightly extended standard model of elementary particles, right handed (or sterile) neutrinos of mass $\lesssim 20 \mathrm{keV}$ are not in contrast with cosmological constraints (see also Shi \& Fuller 1999). However, this issue cannot be considered firmly established since this model is constrained by an astrophysical bound on $\nu_{\mathrm{s}} \rightarrow \nu \gamma$ (see e.g. Dress $\&$ Wright 2000). Alternatively, in contrast with the standard cosmology, some authors (Viollier 1994; Bilić et al. 2000; Lindebaum et al. 1999) have proposed a scenario according to which the universe become heavy neutrino matter-dominated 22 days after the Big Bang at temperature $\sim 1 \mathrm{keV}$. From that time on, the evolution of the universe differed substantially from the standard cosmology results since the universe will undergo a gravitational phase transition leading to super-massive neutrino systems with masses close to the Oppenheimer-Volkoff limit $\simeq 3 \times 10^{9} M_{\odot}$ (Lindebaum et al. 1999 and references therein). At this stage, annihilation of heavy neutrinos into non-standard light bosons may take place, thereby reducing the neutrino number density inside neutrino systems.

However, well aware that in the framework of standard cosmology the heavy neutrino hypothesis meets with difficulties, in the present paper we focus on a set of independent astrophysical constraints on the neutrino mass that can be derived from the observational data towards the galactic center. Obviously, further theoretical analysis is necessary in order to clarify if heavy neutrinos really exist and cluster in the galactic centers. Anyway, the next generation of X-ray and $\gamma$-ray satellites, with improved sensitivity and angular resolution, will allow us to definitely confirm or exclude the presence of a massive neutrino ball at the galactic center. 
The outline of the paper is the following: in Sect. 2 we describe the adopted neutrino ball model, then we consider in Sect. 3 a set of astrophysical constraints which can be put on the neutrino ball parameters. In Sect. 4, we investigate the observational signatures from this exotic object at the Galactic Center. Our main conclusions are summarized in Sect. 5 .

\section{The neutrino ball model}

The gravitational equilibrium of a fully degenerate system of fermions is well known since Chandrasekhar (1939), who showed that equilibrium configurations do exist if the total number of particles is less than the critical value $N_{\text {crit }} \simeq\left(m_{\text {Planck }} / m_{\nu}\right)^{3}$. Here $m_{\text {Planck }} \simeq(\hbar c / G)^{1 / 2}$ is the Planck mass. However, it has been stressed by several authors that in the standard cosmology a degeneracy value near zero (corresponding to the semi-degenerate case) should be expected for neutrinos produced in the early universe (see e.g. Dolgov \& Zel'dovich 1981). When the gravitational configurations of semi-degenerate systems of fermions are calculated, a spatial divergence appears and the solutions are not finite in mass and radius, as in the case of isothermal systems obeying the classical statistics (Gao \& Ruffini 1980). A solution to this problem has been proposed by Ruffini \& Stella (1983) on the basis of the early work of King (1966). These works introduce a distribution function modified with an energy cutoff in phase space and allows one to obtain self-gravitating systems limited in extension since the velocity of the particles at any point in the system has to be lower than the escape velocity. In the case of spherical symmetry and within the non-relativistic approximation (see below), this escape velocity is given in terms of the gravitational potential $V(r)$ by

$v_{\mathrm{e}}^{2}(r)=-2 V(r)$,

where $V(r)$ is fixed to be zero at the boundary $R$ of the system.

Massive neutrinos are considered to be collisionless and are described in the momentum space by the distribution function (Ruffini \& Stella 1983)

$$
\begin{cases}\mathrm{d} n(r)=\frac{g}{h^{3}} \frac{1-\mathrm{e}^{\left(\epsilon-\epsilon_{\mathrm{C}}(r)\right) / k T}}{\mathrm{e}^{(\epsilon-\mu(r)) / k T}+1} \mathrm{~d}^{3} p(\epsilon) & \text { for } \epsilon \leq \epsilon_{\mathrm{c}}(r) \\ \mathrm{d} n(r)=0 & \text { for } \epsilon>\epsilon_{\mathrm{c}}(r),\end{cases}
$$

where $\epsilon_{\mathrm{c}}(r)$ is the energy cutoff for neutrinos, $g=2 s_{\nu}+1$ the spin multiplicity of quantum states, $\mu(r)$ the chemical potential and $T$ the fermionic thermodynamic temperature assumed to be constant. This distribution function generalizes, to semi-degenerate systems of fermions, the distribution function introduced by King (1966) for systems of classical particles (for which $\mu(r) \rightarrow-\infty$ ). At the same time it includes the usual Fermi-Dirac statistics, which are recovered from Eq. (2) in the limit $\mu(r) \rightarrow+\infty$ and $\epsilon_{\mathrm{c}}(r) \rightarrow+\infty$.
In view of the application of the distribution function in Eq. (2) to the neutrino ball at the Galactic Center, we restrict our attention to the non-relativistic regime because of the densities involved in this structure. With the approximation $\epsilon=m_{\nu} v^{2} / 2$ and $k T<<m_{\nu} c^{2}$, after integration on $\mathrm{d}^{3} p$, one gets the mass density $\rho(r)$ and the pressure $p(r)$ as a function of the radial coordinate $r$

$\rho(r)=\frac{2 \pi g m_{\nu}^{4}}{h^{3} j^{3}} \int_{0}^{W(r)} \frac{1-\mathrm{e}^{x-W(r)}}{\mathrm{e}^{x-\theta(r)}+1} x^{1 / 2} \mathrm{~d} x$,

$p(r)=\frac{2}{3} \frac{\pi g m_{\nu}^{4}}{h^{3} j^{5}} \int_{0}^{W(r)} \frac{1-\mathrm{e}^{x-W(r)}}{\mathrm{e}^{x-\theta(r)}+1} x^{3 / 2} \mathrm{~d} x$,

where

$x=j^{2} v^{2}, \quad j^{2}=\frac{m_{\nu}}{2 k T}$

and the quantities $W(r)$ and $\theta(r)$ are the energy-cutoff and degeneracy parameter defined by

$W(r)=\frac{\epsilon_{\mathrm{c}}(r)}{k T} \quad$ and $\quad \theta(r)=\frac{\mu(r)}{k T}$.

We note that in the fully degenerate limit, being $\theta(r) \gg 1$ and $W(r) \gg 1$, Eqs. (3) and (4) give the usual density and pressure of a degenerate Fermi gas (Chandrasekhar 1939)

$[\rho(r)]_{\mathrm{deg}}=\frac{4 \pi g m_{\nu}^{4}}{3 h^{3}}\left(\frac{2 \mu(r)}{m_{\nu}}\right)^{3 / 2}$,
$[p(r)]_{\mathrm{deg}}=\frac{4 \pi g m_{\nu}^{4}}{15 h^{3}}\left(\frac{2 \mu(r)}{m_{\nu}}\right)^{5 / 2}$.

Density and pressure in Eqs. (3) and (4) depend on four parameters: the mass of the particle $m_{\nu}$, the temperature parameter $j$, the energy cutoff parameter $W(r)$ and the degeneracy factor $\theta(r)$. However, the assumed condition of thermal equilibrium in the presence of a gravitational field for the neutrino ball implies the following relation between $\theta(r)$ and $W(r)$ (Ingrosso et al. 1992)

$\theta(r)=W(r)+\theta_{\mathrm{R}}$,

where $\theta_{\mathrm{R}} \leq 0$ is the degeneracy evaluated at the system's surface.

Assuming spherical symmetry, within the nonrelativistic limit, the equations governing the gravitational equilibrium of the self-gravitating systems are

$\frac{\mathrm{d} p}{\mathrm{~d} r}=-\frac{G M(r) \rho}{r^{2}}, \quad \frac{\mathrm{d} M}{\mathrm{~d} r}=4 \pi r^{2} \rho$,

which can be recasted in the form

$\frac{\mathrm{d} W}{\mathrm{~d} r}=-2 j^{2} \frac{G M(r)}{r^{2}}$,

from which it follows that

$\frac{\mathrm{d}^{2} W}{\mathrm{~d} \xi^{2}}+\frac{2}{\xi} \frac{\mathrm{d} W}{\mathrm{~d} \xi}=-8 \pi G j^{2} r_{0}^{2} \rho$ 
where $\xi=r / r_{0}$ is a dimensionless radius expressed in terms of the "core radius"

$r_{0}^{2}=\frac{9}{8 \pi G \rho(0) j^{2}}$.

We note that the particle velocity dispersion obeys the relation

$<v^{2}(r)>=\frac{3 p(r)}{\rho(r) j^{2}}$,

which in the classical limit reduces to the Boltzmann relation $\left\langle v^{2}(r)\right\rangle=3 /\left(2 j^{2}\right)$, while in the fully degenerate case reads $\left\langle v^{2}(r)\right\rangle=3 W(r) /\left(5 j^{2}\right)$.

Equation (12) has to be integrated with the boundary conditions $W(0)=W_{0}$ and $W^{\prime}(0)=0$ from the center of the configuration to the surface at which $W(R)=0$. Clearly, Eq. (12), through the definition of the density $\rho(r)$, also depends on the degeneracy parameter at the surface of the configuration $\theta_{\mathrm{R}}$, while the dependence on the parameters $m_{\nu}$ and $j$ disappears by the definition of $r_{0}$. The radius $R$ and the total mass $M$ of the system are given by

$$
R=r_{0} \xi_{\mathrm{R}} \quad \text { and } \quad M=\frac{r_{0}}{2 G j^{2}}\left(-\xi^{2} \frac{\mathrm{d} W}{\mathrm{~d} \xi}\right)_{\xi=\xi_{\mathrm{R}}},
$$

where $\xi_{\mathrm{R}}$ is the value of the radial coordinate at which $W\left(\xi_{\mathrm{R}}\right)=0$ and $\rho\left(\xi_{\mathrm{R}}\right)=0$.

A detailed analysis of the numerical solutions of Eq. (12) has been performed by Ruffini \& Stella (1983) and Ingrosso et al. (1992), showing that simple scaling relations between $m_{\nu}, M$ and $R$ may be found both in the classical and in the degenerate cases. In fact, in the classical limit $\theta_{\mathrm{R}} \rightarrow-\infty$, one gets

$m_{\nu} \propto R^{-3 / 8} \mathrm{e}^{-\theta_{\mathrm{R}} / 4} M^{-1 / 8}$.

In the degenerate limit the dependence of $m_{\nu}$ on the parameter $\theta_{\mathrm{R}}$ disappears so that the scale law is

$m_{\nu} \propto R^{-3 / 8} M^{-1 / 8}$,

corresponding to the well-known Chandrasekhar's (1939) scaling laws. The typical relationship between the ball radius and the constituting neutrino mass is shown in Fig. 1 and it will be discussed in the following section.

\section{Astrophysical constraints}

The aim of this section is to determine the constraints that current astrophysical observations can put on the physical parameters of a possible neutrino ball at the Galactic Center, described by the model outlined in Sect. 2. These constraints are the consequence both of the available astronomical observations, like star dynamics within $1 \mathrm{pc}$ from the Galactic Center and the SgrA* luminosity observed in all wavelenghts, and of the theoretical mass limit for stable configurations of fermions.

First of all, it is well known that the two dimensional positions and velocities measured for stars in the inner

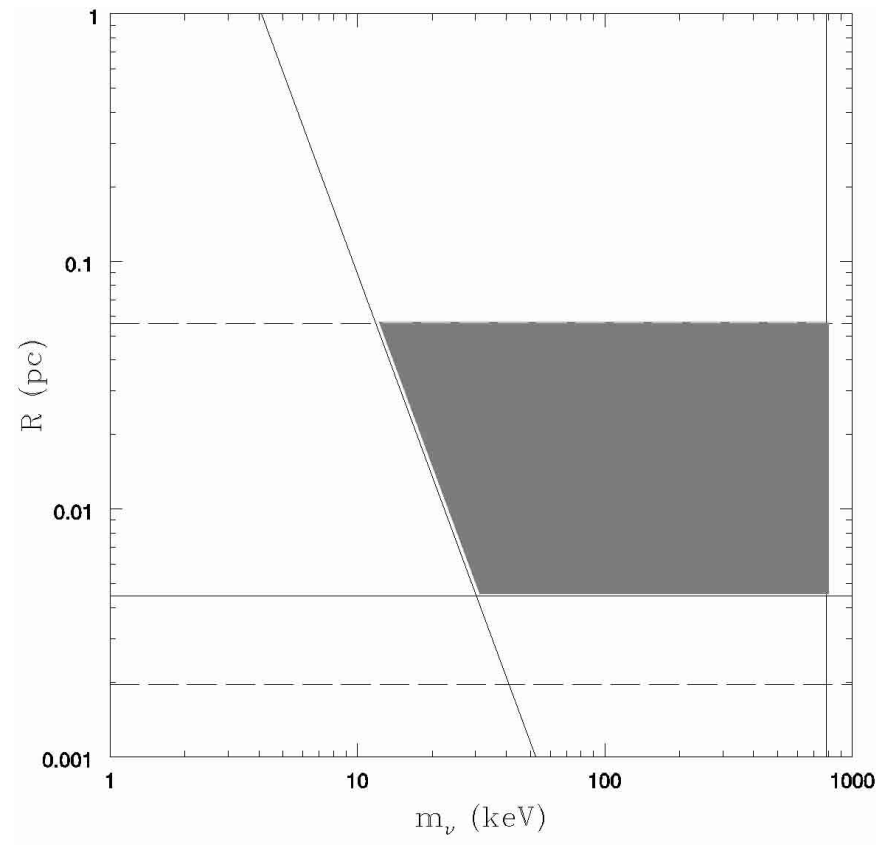

Fig. 1. The neutrino ball radius $R$ is reported as a function of the constituting neutrino mass $m_{\nu}$. The neutrino ball mass has been assumed to be $M=(2.6 \pm 0.2) \times 10^{6} M_{\odot}$. The oblique dashed line corresponds to the full degenerate models. The vertical continuous line represents the Chandrasekhar limit. The two horizontal dashed lines bound the models satisfying the observed luminosity towards SgrA*. The models above the horizontal solid line have $T_{\mathrm{ev}} \geq T_{\mathrm{H}}$. Details are throughout the text.

$6^{\prime \prime} \times 6^{\prime \prime}(0.23 \times 0.23 \mathrm{pc})$ provide excellent constraints on the matter distribution at the galactic center. With the assumption that stars are gravitationally bound by the central gravitational potential, Bahcall \& Tremaine (1981) proposed to use a projected mass estimator which, for the case of star isotropic orbits, is given by

$M=\frac{16}{\pi G}<v^{2}(b) b>$

where $v(b)$ and $b$ represent the star projected velocity and distance from $\mathrm{SgrA}^{*}$, respectively. For the Galactic Center data the estimator above gives, for the amount of matter contained within $0.015 \mathrm{pc}$, the value $M=(2.6 \pm 0.2) \times$ $10^{6} M_{\odot}$ (Ghez et al. 1998).

As far as the SgrA* luminosity is concerned, Melia (1992) showed that observations of stellar winds and gas flows near $\mathrm{SgrA}^{*}$, coupled with the above estimate of the SBH mass, imply a minimum mass accretion rate $\dot{M} \simeq$ $10^{-6} M_{\odot} \mathrm{yr}^{-1}$ (Genzel et al. 1994). This estimate, for a standard thin accretion disk around the SBH, would imply a total luminosity $L \geq 10^{41} \mathrm{erg} \mathrm{s}^{-1}$ with a peak emission in the X-ray band. On the contrary, the total luminosity observed from radio to $\gamma$-ray wavelengths is below $10^{37} \mathrm{erg} \mathrm{s}^{-1}$, peaked in the near infrared region corresponding to a photon energy $E_{\gamma} \simeq 5 \times 10^{-5} \mathrm{keV}$ (Narayan et al. 1998).

In the neutrino ball scenario, assuming that the star dynamics around $\operatorname{SgrA}^{*}$ is accounted for by the 
galactic neutrino ball gravitational potential, we need that the mass enclosed within about $10^{-2} \mathrm{pc}$ is $M \simeq$ $2.6 \times 10^{6} M_{\odot}$. This condition, as it is evident from Eqs. (16) and (17), allows us to determine the neutrino mass as a function of the ball radius in the case of degenerate systems, and of both the ball radius and degeneracy parameter $\theta_{\mathrm{R}}$ in the case of semi-degenerate ones. This is shown in Fig. 1 where the oblique dashed line corresponds to the fully degenerate systems. The models on the right part with respect to this line are models with decreasing values of the degeneracy parameter $\theta_{\mathrm{R}}$ and increasing values of the neutrino mass $m_{\nu}$.

An upper limit to the constituting neutrino mass $m_{\nu}$ does exist as a consequence of General Relativity. In fact, it is well known (see e.g. Shapiro \& Teukolsky 1983) that the balance between the gravitational force and the degeneracy pressure leads to stable configurations of fermions until the number of particles composing the system does not exceed the critical value given by

$\left(N_{\nu}\right)_{\max } \simeq\left(\frac{\hbar c}{G}\right)^{3 / 2} m_{\nu}^{-3}$.

In this way, the maximum neutrino mass allowed by the General Relativity can be estimated as a function of the total mass by

$\left(m_{\nu}\right)_{\max } \simeq 787\left(\frac{2.6 \times 10^{6} M_{\odot}}{M}\right)^{\frac{1}{2}} \mathrm{keV}$,

which, for the assumed neutrino ball mass, entails $m_{\nu, \max } \simeq 787 \mathrm{keV}$. This limit corresponds to the vertical solid line in Fig. 1.

In addition to the above dynamical constraint, the SgrA* luminosity also has to be consistent with the ball parameters $M, R$ and $m_{\nu}$. We assume that the luminosity observed from the Galactic Center is the result of the accretion of the surrounding gas on the neutrino ball. In this case, for a spherical inflow, the energy of the emitted thermalized photons is given by (Shapiro \& Teukolsky 1983)

$E_{\gamma} \simeq 10^{-5} R_{16}^{-1 / 2} L_{37}^{1 / 4} \mathrm{keV}$,

where $R_{16}$ and $L_{37}$ represent the neutrino ball radius and the total luminosity in units of $10^{16} \mathrm{~cm}$ and $10^{37} \mathrm{erg} \mathrm{s}^{-1}$, respectively.

Therefore, for an observed SgrA* luminosity $L \simeq$ $10^{37} \mathrm{erg} \mathrm{s}^{-1}$, and for a photon energy $E_{\gamma} \simeq(5 \pm 1) \times$ $10^{-5} \mathrm{keV}$, the previous equation entails a set of acceptable neutrino ball models corresponding to the region between the two horizontal dashed lines in Fig. 1. As one can see, the lower limit to the neutrino mass is $m_{\nu} \simeq 11 \mathrm{keV}$ corresponding to the maximum acceptable neutrino ball radius $R \simeq 5.5 \times 10^{-2} \mathrm{pc}$. We note also that the mass enclosed within 0.015 pc is $2.42 \times 10^{6} M_{\odot}$, in agreement with the observational constraints.

An additional constraint on the allowed neutrino ball parameters $R$ and $m_{\nu}$ derives by requiring that the evaporation time-scale of the system is greater than the Hubble time $T_{\mathrm{H}} \simeq 1.4 \times 10^{10} \mathrm{yrs}$. In fact, by considering the interaction among neutrinos and anti-neutrinos of the ball itself via the following reaction channels (Boehm \& Vogel 1987)

$\nu_{\tau}+\bar{\nu}_{\tau} \rightarrow \nu_{\mathrm{e}}+\bar{\nu}_{\mathrm{e}}$

$\nu_{\tau}+\bar{\nu}_{\tau} \rightarrow \nu_{\mu}+\bar{\nu}_{\mu}$,

the evaporation time-scale can be defined as

$T_{\mathrm{ev}} \simeq \frac{1}{n_{\nu}<v_{\nu}>\sigma_{\nu \nu}}$

where $\sigma_{\nu \nu} \simeq G_{\mathrm{f}}^{2} m_{\nu_{\tau}}^{2} / 2 \pi \hbar^{4} g\left(G_{\mathrm{f}}\right.$ is the Fermi coupling constant) is the process cross section (Holdom \& Malaney 1994) and the neutrino mean velocity $\left\langle v_{\nu}\right\rangle$ is given by Eq. (14). By requiring that $T_{\mathrm{ev}} \geq T_{\mathrm{H}}$ we obtain that the allowed models are those above the horizontal solid line in Fig. 1 corresponding to a minimum neutrino ball radius of $4.3 \times 10^{-3} \mathrm{pc}$.

At this stage, the allowed neutrino ball parameters are represented by the grey region in Fig. 1. In the next section, the allowed region will be further reduced by considering the available observations both in the X-ray and $\gamma$-ray energy bands towards SgrA*.

\section{Search for galactic neutrino ball signatures}

In order to study the influence of a neutrino ball at the Galaxy Center, we investigate the observable signatures that should be produced $i$ ) in the interaction of incoming high energy neutrinos (or anti-neutrinos) with antineutrinos (or neutrinos) composing the ball, and ii) in the interaction between neutrinos and anti-neutrinos in the ball itself.

Let us first consider the case $i$ ). The existence of an high energy $\mu$ neutrino (and/or anti-neutrino) flux is a theoretical consequence of the decay of charged pions produced in high-energy $p N$ interactions. From Cosmic Ray observations on Earth, Waxman \& Bahcall (1998) derive an upper bound to the high energy neutrino flux given by

$\frac{\mathrm{d} N_{\nu_{\mu}}}{\mathrm{d} E_{\nu_{\mu}}} \simeq 2 \times 10^{-8}\left(\frac{E_{\nu_{\mu}}}{\mathrm{GeV}}\right)^{-2} \frac{\text { neutrinos }}{\mathrm{cm}^{2} \mathrm{~s} \mathrm{sr} \mathrm{GeV}}$

where $E_{\nu_{\mu}}$ represents the energy of the incident $\mu$ neutrinos. The previous relation holds for $E_{\gamma} \geq 10^{3} \mathrm{GeV}$. In the framework of the Standard Model for weak interactions, these particles interact with the massive anti-neutrinos $\bar{\nu}_{\tau}$ (or neutrinos $\nu_{\tau}$ ) composing the ball, giving rise to a photon flux on Earth, as a consequence of the final neutral pion decays (Fargion et al. 1999). Indeed, for a $\nu_{\mu} \bar{\nu}_{\tau}$ (and charge conjugated $\bar{\nu}_{\mu} \nu_{\tau}$ ) interaction via the $W$ exchange in the $t$-channel, the cross section is given by (Fargion et al. 1999)

$\sigma(s) \simeq 108.5 \frac{A}{s}\left\{1+\frac{m_{W}^{2}}{s} \times\left[2-\frac{s-B}{A} \ln \left(\frac{B+A}{B-A}\right)\right]\right\} \mathrm{pb}$,

where $m_{W}$ is the $W$ boson mass, $\sqrt{s}$ is the center of mass energy which - in the relativistic limit reads out to be 


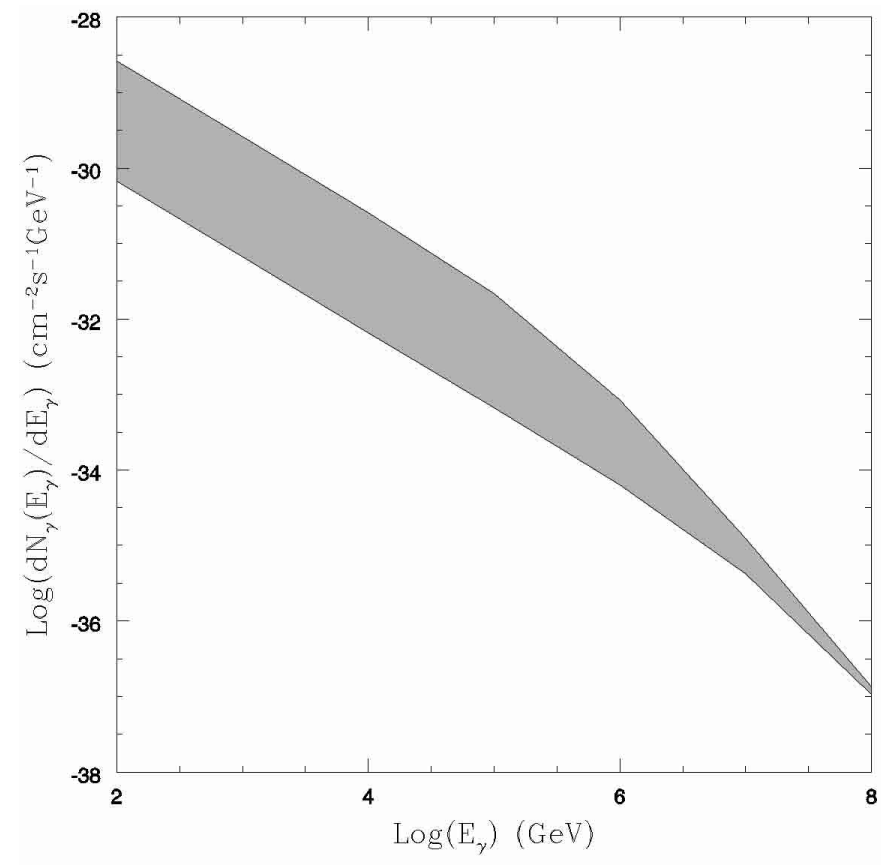

Fig. 2. The photon flux $\mathrm{d} N_{\gamma}\left(E_{\gamma}\right) / \mathrm{d} E_{\gamma}$ on Earth, resulting from the interaction of high energy cosmic neutrinos (or antineutrinos) with the anti-neutrinos (or neutrinos) of the ball, is reported as a function of the photon energy $E_{\gamma}$. The dashed region corresponds to different photon fluxes depending on the constituting neutrino mass in the range $20-787 \mathrm{keV}$. Due to the extremely low flux on Earth, this kind of signature cannot be used in order to test for the presence of the neutrino ball at the center of the Galaxy.

$\sqrt{s} \simeq \sqrt{2 m_{\nu_{\tau}} E_{\nu}}-$ and the two functions $A$ and $B$ are defined, respectively, as

$$
\left\{\begin{array}{l}
A=\sqrt{\left[s-\left(m_{\nu_{\tau}}+m_{\nu_{\mu}}\right)^{2}\right]\left[s-\left(m_{\nu_{\tau}}-m_{\nu_{\mu}}\right)^{2}\right]} \\
B=s+2 m_{W}^{2}-m_{\tau}^{2}
\end{array}\right.
$$

where $m_{\nu_{\tau}}$ and $m_{\nu_{\mu}}$ are the neutrino $\nu_{\tau}$ and the neutrino $\nu_{\mu}$ masses, respectively. From the $t$-channel reaction chain for final photon production, it is easy to observe that the energy of each incident neutrino is a multiple $\eta$ of the energy of the produced photon $\left(E_{\nu_{\mu}} \simeq \eta E_{\gamma}\right)$ and that each step of the chain occurs with probability $P_{i}$ (Fargion et al. 1999).

The photon flux on Earth obviously depends on the neutrino ball parameter, i.e. the radius $R$ and the neutrino number density $n_{\nu_{\tau}}(r) \simeq \rho(r) / m_{\nu_{\tau}}$. In this way, the photons flux at energy $E_{\gamma}$ is estimated to be

$\frac{\mathrm{d} N_{\gamma}}{\mathrm{d} E_{\gamma}} \simeq \int_{0}^{R} \frac{\mathrm{d} N_{\nu_{\mu}}}{\mathrm{d} E_{\nu_{\mu}}}\left(\frac{r}{D}\right)^{2} \mathrm{e}^{\sigma(s) n_{\nu_{\tau}}(r-R)} \sigma(s) n_{\nu_{\tau}} \prod_{i} P_{i} \mathrm{~d} r$

where $D \simeq 8.5 \mathrm{Kpc}$ is the Earth distance from the Galactic Center. In Fig. 2 we show the flux $\mathrm{d} N_{\gamma} / \mathrm{d} E_{\gamma}$ for different values of $E_{\gamma}$ and $m_{\nu_{\tau}} \gtrsim 20 \mathrm{keV}$, assuming for the neutrino ball mass and radius the values $M \simeq 2.6 \times 10^{6} M_{\odot}$ and $R=10^{-2} \mathrm{pc}$, respectively. In all cases, the $\gamma$-ray flux on Earth is too low to be measured by the actual instrumentation, leaving open the problem of discriminating the Galactic Center dark object by using this kind of signature.

If a neutrino ball really exists at the Galactic Center, other possible characteristic signatures come from the interaction among neutrinos and anti-neutrinos of the ball itself via the radiative reaction channel and by the $\tau$ neutrino decay (Boehm \& Vogel 1987)
(a) $\nu_{\tau}+\bar{\nu}_{\tau} \rightarrow \gamma+\gamma$
(b) $\nu_{\tau} \rightarrow \nu_{\mathrm{e}}+\gamma$.

Following Viollier \& Trautmann (1993), we assume that the reaction channel $(a)$ produces photons at energy $E_{\gamma}=$ $m_{\nu} c^{2}$ giving rise to an emission line with luminosity

$$
L_{\gamma}^{a} \simeq 4 \times 10^{25}\left(\frac{M}{2.6 \times 10^{6} M_{\odot}}\right)^{3} \times\left(\frac{m_{\nu_{\tau}} c^{2}}{14.5 \mathrm{keV}}\right)^{9} \mathrm{erg} \mathrm{s}^{-1} .
$$

Further, neglecting X-ray absorption along the line of sight to the Galactic Center ${ }^{2}$, the corresponding photons flux on Earth turns out to be

$$
\begin{aligned}
\Phi_{\gamma}^{a} \simeq & 4.5 \times 10^{-13}\left(\frac{M}{2.6 \times 10^{6} M_{\odot}}\right)^{3} \\
& \times\left(\frac{m_{\nu_{\tau}} c^{2}}{14.5 \mathrm{keV}}\right)^{8} \mathrm{~cm}^{-2} \mathrm{~s}^{-1} .
\end{aligned}
$$

Another possible neutrino ball signature comes from the (b) reaction channel in (28). In fact, as a consequence of the standard electroweak interaction theory, heavy neutrinos may decay into lighter neutrino species trough the emission of a photon producing an X-ray emission line at energy $E_{\gamma}=m_{\nu_{\tau}} c^{2} / 2$. The corresponding photon luminosity is

$L_{\gamma}^{b} \simeq \frac{M c^{2}}{2 T_{\mathrm{D}}}$

where the radiative decay constant $T_{\mathrm{D}}$ is given by (Viollier et al. 1992)

$T_{\mathrm{D}}^{-1} \leq 3 \times 10^{-19}\left(\frac{m_{\nu_{\tau}} c^{2}}{14.5 \mathrm{keV}}\right)^{5} \mathrm{yr}^{-1}$.

${ }^{2}$ Generally, in order to evaluate the photon flux on Earth we have also to consider the interstellar absorption due to the interaction of photons with the interstellar medium protons. In this way the photon flux on Earth should be

$\Phi_{\gamma} \simeq \frac{L_{\gamma}}{4 \pi D^{2}} \mathrm{e}^{-\tau}$,

where the total optical depth $\tau$ is the sum of the two terms $\tau_{1}=\sigma_{\gamma \mathrm{p}} n_{1, \mathrm{p}} D_{1}$ and $\tau_{2}=\sigma_{\gamma \mathrm{p}} n_{2, \mathrm{p}} D_{2}$. The cross section for $\gamma-\mathrm{p}$ interaction is given by (Morrison \& McCammon 1983)

$\sigma_{\gamma \mathrm{p}} \simeq 2.3 \times 10^{-22}\left(E_{\gamma} / \mathrm{keV}\right)^{-8 / 3} \mathrm{~cm}^{2}$

and the proton number densities $n_{1, \mathrm{p}}$ and $n_{2, \mathrm{p}}$ are assumed to be $n_{1, \mathrm{p}} \simeq 100 \mathrm{~cm}^{-3}$ within $D_{1} \simeq 100 \mathrm{pc}$ from $\mathrm{SgrA}^{*}$ (see also Zane et al. 1996) and $n_{2, \mathrm{p}} \simeq 1 \mathrm{~cm}^{-3}$ from the central region to Earth at the distance $D_{2} \simeq 8.5 \mathrm{kpc}$, respectively. As we will see, in the case of interest $\left(E_{\gamma} \geq 10 \mathrm{keV}\right)$ the absorption turns out to be negligible. 
Consequently, the luminosity entails

$L_{\gamma}^{b} \leq 2.3 \times 10^{34}\left(\frac{M}{2.6 \times 10^{6} M_{\odot}}\right) \times\left(\frac{m_{\nu_{\tau}} c^{2}}{14.5 \mathrm{keV}}\right)^{5} \mathrm{erg} \mathrm{s}^{-1}$,

corresponding to a photon flux on Earth

$$
\begin{aligned}
\Phi_{\gamma}^{b} \leq & 1.2 \times 10^{-4}\left(\frac{M}{2.6 \times 10^{6} M_{\odot}}\right) \\
& \times\left(\frac{m_{\nu_{\tau}} c^{2}}{14.5 \mathrm{keV}}\right){ }^{4} \mathrm{~cm}^{-2} \mathrm{~s}^{-1} .
\end{aligned}
$$

Let us now compare this expected photon flux with the presently available data. In the energy range of interest (10 keV $\left.\lesssim E_{\gamma}<787 \mathrm{keV}\right)$, data have been collected mainly by the instruments ART-P on board of GRANAT and OSSE on CGRO.

ART-P made detailed observations towards the Galactic Center region in the energy band $3 \mathrm{keV}-30 \mathrm{keV}$ (Pavlinsky et al. 1994) and in particular towards SgrA* with exposure time $\simeq 164000 \mathrm{~s}$. The derived photon spectrum is well described by a power law model with index $\alpha=-1.6 \pm 0.1$ and the measured average flux, in the $3-20 \mathrm{keV}$ energy band, is $8.8 \times 10^{-3}$ photons $\mathrm{cm}^{-2} \mathrm{~s}^{-1}$. The absence of lines and/or of other particular features is clear from Fig. 6 on Pavlinsky et al. (1994).

The OSSE instrument on the CGRO satellite has also observed the Galactic Center in the energy range $30 \mathrm{keV}-$ $1 \mathrm{MeV}$ with an exposure time of about one day (Smith et al. 1995). The observed $\operatorname{SgrA}^{*}$ spectrum can be well fitted by a power law model with index $\alpha=-2.1 \pm 0.1$ and with average flux, in the $30-600 \mathrm{keV}$ energy band, of $9.7 \times 10^{-5}$ photons $\mathrm{cm}^{-2} \mathrm{~s}^{-1}$ (Smith et al. 1995). It is important to note that in the OSSE spectrum of the Galactic Center only two structures were observed, i.e. the emission line at $511 \mathrm{keV}$, corresponding to the electronpositron annihilation radiation, and the emission feature at $170 \mathrm{keV}$ which is interpreted as the Compton backscattered $511 \mathrm{keV}$ radiation (Smith et al. 1995).

A flux $\Phi_{\gamma}$, due to an emission line at energy $E_{\gamma}$, is detectable with $k \sigma$ statistical detection threshold if

$\Phi_{\gamma}>k \sqrt{\frac{\Phi_{\gamma}^{\mathrm{obs}}\left(E_{\gamma}\right)}{T_{\mathrm{obs}}}}$,

where $T_{\text {obs }}$ is the observation time and

$\Phi_{\gamma}^{\mathrm{obs}}\left(E_{\gamma}\right)=\int_{E_{\gamma}-\Delta E}^{E_{\gamma}+\Delta E} \frac{\mathrm{d} N}{\mathrm{~d} E} \mathrm{~d} E$

is the observed flux on Earth. Here $\mathrm{d} N / \mathrm{d} E$ is the photon spectrum as observed by the ART-P and OSSE satellites and $2 \Delta E$ is the instrument spectral resolution (see Pavlinsky et al. 1994; Molkov et al. 1999; Johnson et al. 1993).

Clearly, due to the neutrino mass range $11 \mathrm{keV} \lesssim m_{\nu} \lesssim$ $787 \mathrm{keV}$ (obtained from the analysis in Fig. 1), the photon flux $\Phi_{\gamma}^{b}$ in Eq. (36) is the most favorite signature for direct observation. Thus, by setting $\Phi_{\gamma}\left(E_{\gamma}\right) \equiv \Phi_{\gamma}^{b}\left(m_{\nu} c^{2} / 2\right)$, the two hand sides of Eq. (37) are plotted in Fig. 3 as a function of the line energy $E_{\gamma}$. Inspection of this figure allows

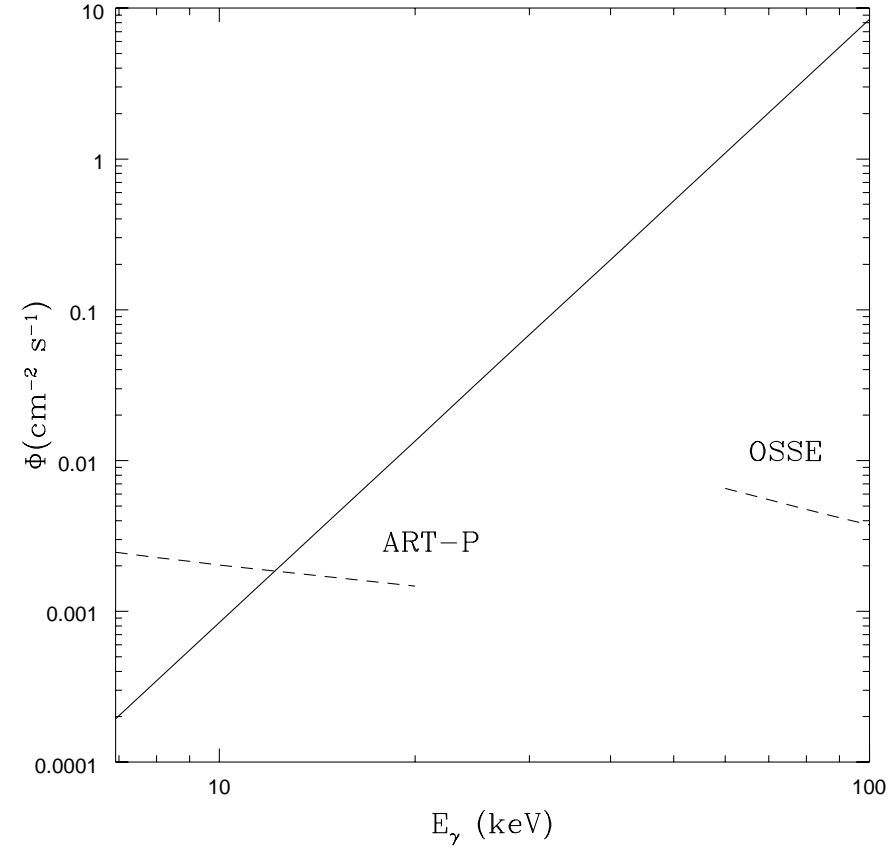

Fig. 3. The photon flux on Earth, produced by the decay of massive neutrinos into lighter species throughout a photon emission, is reported for different constituting neutrino mass. The neutrino ball mass has been assumed to be $M=$ $(2.6 \pm 0.2) \times 10^{6} M_{\odot}$. The dashed lines show the threshold flux necessary to see a line at energy $E_{\gamma}$ within $5 \sigma$ statistical detection confidence level for the instruments ART-P and OSSE. The continuous line gives the expected photon flux in a line at energy $E_{\gamma}=m_{\nu} c^{2} / 2$ (for details see text).

us to reject neutrino ball models corresponding to neutrinos with mass $m_{\nu} \gtrsim 24 \mathrm{kev}$ since they are expected to imply emission lines which should have been detected by ART-P. Consequently, the permitted neutrino mass range reduces to $11 \mathrm{keV} \lesssim m_{\nu} \lesssim 24 \mathrm{keV}$.

\section{Discussion and conclusion}

In this paper, we investigate the possibility that the Galactic Center hosts a massive neutrino ball of total mass $M \simeq 2.6 \times 10^{6} M_{\odot}$. The existence of such an object, under particular circumstances, should justify the low luminosity, from radio to $\gamma$-rays, observed in the direction of $\operatorname{Sgr} A^{*}$.

We build up models for the neutrino ball by studying the gravitational equilibrium of a semi-degenerate fermion gas. Density and pressure within the ball are defined by adopting a formalism based on a distribution function in phase space, which allows us to consider neutrinos with a degeneracy degree varying from the center to the border of the system. Limiting cases are the fully degenerate fermion systems (which are represented by the oblique dashed line in Fig. 1) and the classical isothermal spheres well known in the literature. The local balance between gravitational force and pressure gradient leads to stable configurations if the number of neutrinos (and/or antineutrinos) does not exceed the critical value in Eq. (19). 
This fact, for a total ball mass $M \simeq 2.6 \times 10^{6} M_{\odot}$, allows us to put an upper limit to the neutrino mass $m_{\nu} \lesssim 787 \mathrm{keV}$. This limit is represented in Fig. 1 by the vertical solid line. Acceptable neutrino ball models in Fig. 1 are those between the oblique dashed line and the vertical solid one, having decreasing degeneracy with increasing neutrino mass $m_{\nu}$.

By requiring, moreover, that the observed luminosity towards SgrA* comes from the accretion process on the neutrino ball and that the evaporation time scale of the ball is longer than the Hubble time, the allowed models are those on the grey region in Fig. 1. Correspondingly, we get that $11 \mathrm{keV} \lesssim m_{\nu} \lesssim 787 \mathrm{keV}$.

The above neutrino mass range can be further reduced by studying the photon flux on Earth due to $i$ ) interaction of incoming ultra high energy neutrinos (or anti-neutrinos) with anti-neutrinos (or neutrinos) composing the ball, and ii) interaction between neutrinos and anti-neutrinos in the ball itself (28 a) and $\tau$ neutrino decay (28 b). Investigation of such effects gives us the opportunity to test the model itself by comparing the neutrino ball signature with the available satellite observations. In particular, the neutrino decay reaction $\nu_{\tau} \rightarrow \nu_{\mathrm{e}}+\gamma$ gives rise to an emission line at energy $m_{\nu_{\tau}} c^{2} / 2$. However, a detailed analysis of the observed spectrum towards the Galactic Center allows us to exclude such a signal for a constituting neutrino mass $m_{\nu} \gtrsim 24 \mathrm{keV}$ (see Fig. 3). Therefore, present observations do not allow us to exclude the existence of a neutrino ball at the Galactic Center with mass $M \simeq 2.6 \times 10^{6} M_{\odot}$ if the constituting neutrino mass $m_{\nu}$ is in the range $11 \mathrm{keV} \lesssim m_{\nu} \lesssim 24 \mathrm{keV}$. The next generation of X-ray satellites, like XEUS (XEUS home-page 2000) and Constellation-X (Constellation-X home-page 2000), with improved sensitivity and angular resolution, will be able to definitively exclude or confirm the existence of a neutrino ball with constituting particle mass in the above range.

Acknowledgements. We thank Dr. Daniele Montanino for interesting discussions.

\section{References}

Agol, E. 2000, ApJ, 538, 121

Bahcall, D. C., \& Tremaine, S. 1981, ApJ, 244, 805

Bilić, N., Lindebaum, R. J., Tupper, G. B., \& Viollier, R. D. [astro-ph/0008230]

Bilić, N., Munyaneza, F., \& Viollier, R. D. 1998, Phys. Rev. D, 59, 024003

Binney, J., \& Tremaine, S. 1987, Galactic Dynamics (Princeton University Press)

Boehm, F., \& Vogel, P. 1987, Physics of massive neutrinos (Cambridge University Press)

Chandrasekhar, S. 1939, An introduction to the Study of Stellar Structure (University of Chicago Press)

Capozziello, S., \& Iovane, G. 1999, Phys. Lett. A, 259, 185

Capozziello, S., Lambiase, G., \& Torres, D. F. 2000, CQG, 17, 3171
Colin, P., Avila-Reese, V., \& Valenzuela, O. 2000, ApJ, 542, 622

Constellation-X Mission 2000, http://constellation.gsfc.nasa.gov/

De Paolis, F., Gurzadyan, V., \& Ingrosso, G. 1996, A\&A, 315, 396

De Paolis, F., Ingrosso, G., \& Nucita, A. A. 2001, A\&A, 366, 1065

Dolgov, A. D., \& Hansen, S. H. 2000 [hep-ph/0103118]

Dolgov, A. D., Hansen, S. H., \& Semikoz, D. V. 1998, Nucl. Phys. B, 524, 6210

Dolgov, A. D., \& Zel'dovich, Ya. B. 1981, Rev. Mod. Phys., 53,1

Dress, M., \& Wright, D. 2000 [hep-ph/0006274]

Fargion, D., Mele, B., \& Salis, A. 1999, ApJ, 517, 725

Gao, J. G., \& Ruffini, R. 1980, Phys. Lett., 97B, 388

Genzel, R., Hollenbach, D., \& Townes, C. H. 1994, Rep. Prog. Phys., 57, 417

Genzel, R., Thatte, N., Krabbe, A., \& Tacconi-Garman, L. E. 1996, ApJ, 472, 153

Ghez, A. M., Klein, B. L., Morris, M., \& Becklin, E. E. 1998, ApJ, 509, 678

Giudice, G. F., Kolbe, E. W., Riotto, A., et al. 2000 [hep-ph/0012317]

Goldwurm, A., Cordier, B., Paul, J., et al. 1994, Nature, 371, 589

Holdom, B., \& Malaney, R. A. 1994, ApJ, 420, L53

Ingrosso, G., Merafina, M., Ruffini, R., \& Strafella, F. 1992, A\&A, 258, 223

Johnson, W. N., Kinzer, R. L., Kurfess, J. D., \& Strickman, M. S. 1993, ApJ, 86, 693

King, I. R. 1966, AJ, 71, 64

Kolb, E. W., \& Turner, M. S. 1990, The Early Universe (Addison-Wesley)

Lindebaum, R. J., Tupper, G. B., \& Viollier, R. D. 1999 [astro-ph/9906004]

Melia, F. 1992, ApJ, 387, L25

Molkov, S. V., Grebenev, S. A., Pavlinsky, M. N., \& Sunyaev, R. A. 1998 [astroph/9903089]

Narayan, R., Mahadevan, R., Grindlay, J. E., et al. 1998, ApJ, 492,554

Morrison, R., \& McCammon, D. 1983, ApJ, 270, 119

Pavlinsky, M. N., Grebenev, S. A., \& Sunyaev, R. A. 1994, ApJ, 425, 110

Ruffini, R., \& Stella, L. 1983, A\&A, 119, 35

Shapiro, S. L., \& Teukolsky, S. A. 1983, Black Holes, white Dwarfs and Neutron Stars (John Wiley \& Sons)

Shi, X., \& Fuller, G. M. 1999, Phys. Rev. Lett., 82, 2832

Smith, D. M., Leventhal, M., Gehrels, N., et al. 1995, ApJ, 443, 117

Torres, D. F., Capozziello, S., \& Lambiase, G. 2000, Phys. Rev. D, 62,104012

Tsiklauri, D., \& Viollier, R. D. 1996, MNRAS, 282, 1299

Tsiklauri, D., \& Viollier, D. 1998a, ApJ, 500, 591

Tsiklauri, D., \& Viollier, D. 1998b, ApJ, 501, 586

Viollier, R. D. 1994, Prog. Part. Nucl. Phys., 32, 51

Viollier, D., \& Trautmann, D. 1994, Phys. Lett. B, 306, 79

Viollier, D., Leimgruber, F. R., \& Trautmann, D. 1992, Phys. Lett. B, 297, 132

Waxman, E., \& Bahcall, J. 1998, Phys. Rev. D, 59

XEUS Collaboration, 2000,

http://astro.estec.esa.nl/SA-general/Projects/XEUS

Zane, S., Turolla, R., \& Treves, A. 1996, ApJ, 471, 248 